\title{
A Passive Transfer Model of the Organ-specific Autoimmune Disease, Bullous Pemphigoid, Using Antibodies Generated against the Hemidesmosomal Antigen, BP180
}

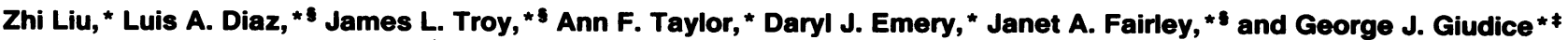 \\ Departments of ${ }^{*}$ Dermatology and ${ }^{\ddagger}$ Biochemistry, Medical College of Wisconsin, Milwaukee, Wisconsin 53226; and the ${ }^{\S}$ Veterans \\ Affairs Medical Center, Milwaukee, Wisconsin 53295
}

\begin{abstract}
Subepidermal blistering associated with the human skin diseases bullous pemphigoid and herpes gestationis has been thought to be an IgG autoantibody-mediated process; however, previous attempts to demonstrate the pathogenicity of patient autoantibodies have been unsuccessful. An immunodominant and potentially pathogenic epitope associated with these blistering diseases has recently been mapped to the extracellular domain of a human epidermal antigen, BP180. Patient autoantibodies that react with this well-defined antigenic site failed to crossreact with the murine form of this autoantigen and thus could not be assayed for pathogenicity in a conventional passive transfer mouse model. As an alternative, rabbit polyclonal antibodies were generated against a segment of the murine BP180 protein homologous with the human BP180 autoantibodyreactive site and were passively transferred into neonatal BALB / $\mathrm{c}$ mice. The injected animals developed a subepidermal blistering disease that closely mimicked bullous pemphigoid and herpes gestationis at the clinical, histological, and immunological levels. Autoantibodies that recognize the human BP180 ectodomain are therefore likely to play an initiatory role in the pathogenesis of bullous pemphigoid and herpes gestationis. ( $J$. Clin. Invest. 1993. 92:2480-2488.) Key words: autoimmunity • hemidesmosome • skin • basement membrane • collagen
\end{abstract}

\section{Introduction}

Bullous pemphigoid (BP) ${ }^{1}$ was described in 1953 by Lever (1) as a subepidermal blistering disease that is seen predominantly in the elderly. In BP patients basal keratinocytes of the epidermis detach from the underlying basement membrane producing tense, fluid-filled vesicles. Similar skin lesions were observed in a pregnancy-associated nonviral disorder, herpes gestationis (HG) (2). Jordon et al. (3) demonstrated by immunofluorescence (IF) techniques that patients with BP exhibit circulating and tissue-bound autoantibodies directed against antigens of the cutaneous basement membrane zone (BMZ). HG patients were also shown to produce comple-

Address correspondence to Dr. George J. Giudice, Department of Dermatology, Medical College of Wisconsin, 8701 Watertown Plank Road, Milwaukee, WI 53226.

Received for publication 19 July 1993 and in revised form $23 \mathrm{Au}$ gust 1993.

1. Abbreviations used in this paper: BMZ, basement membrane zone; BP, bullous pemphigoid; GST, glutathione S-transferase; HG, herpes gestationis; IF, immunofluorescence.

The Journal of Clinical Investigation, Inc.

Volume 92, November 1993, 2480-2488 ment-fixing IgG autoantibodies that exhibit a linear BMZ staining pattern identical to that of BP autoantibodies (4-6).

Several observations supported the concept that the onset and evolution of the skin lesions of BP and HG patients are precipitated by the anti-BMZ autoantibodies. Circulating and BMZ-bound autoantibodies produced by these patients localize to the lamina lucida, the site of blister formation $(7,8) . \mathrm{C}$, detected by IF, binds to the BMZ of lesional skin in both BP and $\mathrm{HG}$ patients (4-6). In almost all HG sera and $>50 \%$ of BP sera the anti-BMZ autoantibodies belong to the IgG1 or IgG3 subclass and avidly fix complement in vitro $(9,10)$. There are well-documented cases of self-limited subepidermal blistering disease in neonates born to mothers with active $\mathrm{HG}$, suggesting transplacental passage of pathogenic autoantibodies $(11,12)$. Plasmapheresis can induce clinical remission in some HG patients $(13,14)$. All of these findings are consistent with a pathogenic mechanism whereby autoantibodies bind to their target antigens in the BMZ, activate complement, and induce subepidermal vesiculation by triggering inflammation.

Two independent antigen/antibody systems associated with BP and HG have recently been characterized at the molecular level (15-18). Both of these autoantigens, designated BP180 and BP230, have been localized to the epidermal hemidesmosome, a cellular structure involved in anchoring basal keratinocytes to the basement membrane (18-20). The BP180 antigen, a $180-\mathrm{kD}$ keratinocyte protein, is of particular interest since it recently has been shown to comprise a unique set of structural and immunological features that make it a prime candidate for harboring pathogenic epitopes. BP180 is a transmembrane protein with a type II orientation (21), i.e., the amino-terminal domain localizes to the intracellular hemidesmosomal plaque (22), while the carboxy-terminal half, made up of a long collagenous tail, projects into the extracellular milieu of the BMZ (23-25). A recent epitope mapping study revealed that $\mathrm{BP}$ and $\mathrm{HG}$ autoantibodies bind a common antigenic site within the major noncollagenous stretch of the BP180 ectodomain (25).

Our laboratory has pioneered the use of passive transfer animal models for the purpose of demonstrating the pathogenic activity of antiepidermal autoantibodies associated with pemphigus vulgaris (26) and pemphigus foliaceus (27), two autoimmune intraepidermal blistering diseases distinct from BP. However, similar approaches failed to demonstrate the pathogenicity of BP autoantibodies $(28,29)$. BP patients' IgG passively transferred into neonatal mice (28) or monkeys (29) exhibited little or no binding to the cutaneous $\mathrm{BMZ}$ of the injected animals and produced no skin lesions, suggesting that the pathogenic BP autoantibodies might not crossreact with the autoantigen homologue of the experimental animal.

In this paper we have used a novel strategy to characterize pathogenically relevant antibodies using tools developed by 
molecular genetic techniques. It was shown that the human BP180 antigen exhibits an unusually high degree of sequence divergence with murine BP180 in the region of the previously defined human antigenic site. Further, autoantibodies that specifically react with this immunodominant epitope on the human BP180 ectodomain lacked the ability to crossreact with the murine form of this protein and therefore could not be tested for pathogenic activity using a conventional murine passive transfer model. As an alternative, rabbit antibodies were generated against a recombinant form of murine BP180 containing the segment homologous with the autoantibody-reactive region of the human antigen. Significantly, passive transfer of these rabbit antibodies into neonatal mice induced cutaneous lesions exhibiting all of the established immunopathological features of BP and HG. The findings of this investigation strongly suggest that anti-BP180 autoantibodies produced in BP and HG patients mediate the subepidermal blister formation characteristic of these diseases.

\section{Methods}

Preparation of recombinant forms of human and murine BP180. A segment of the human BP180 cDNA (nucleotide residue 1627-2671; numbering system taken from reference 21 ) was subcloned into the $3^{\prime}$ end of the Schistosoma japonicum glutathione S-transferase (GST)

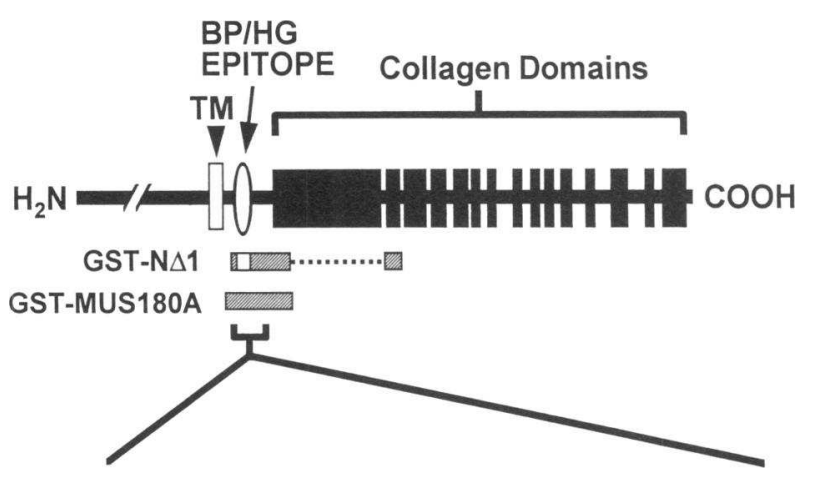

BP/HG EPITOPE

Human: KARVDE LER I R RS I L PYGDSMDR IE KDRL QGMAP

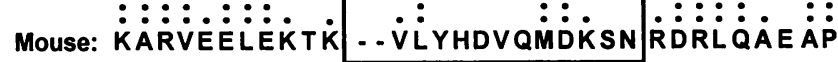

Figure 1. Sequence comparison of human and murine BP180 proteins. The schematic diagram at the top is a structural representation of the BP180 protein based on sequence analysis of the human BP180 cDNA (21). The open rectangle designates the transmembrane domain $(T M)$. The $\mathrm{COOH}$-terminal extracellular region is made up of a series of interrupted collagen triple-helical domains (filled rectangles). The antigenic site recognized by BP and HG autoantibodies (25) is depicted by the open oval. The rectangles located beneath the diagram of the BP180 structure show the sizes and positions of the GST-N $\Delta 1$ and GST-MUS180A recombinant polypeptides relative to the BP180 protein. GST-N $\Delta 1$ contains the BP/HG autoantibodyreactive site (unshaded portion of the rectangle). The dotted line separating the two segments of GST-N $\Delta 1$ designates the portion of BP180 that was deleted from this fusion protein. The bottom portion shows the amino acid sequence alignment of the human and murine forms of BP180 in the region of the epitope recognized by BP and HG autoantibodies (box). Identical residues are designated by double dots and conservative substitutions are marked by a single dot. Note the unusually high degree of sequence divergence in the epitope region (only 3 of 14 residues are identical). gene in the prokaryotic expression vector, pGEX-2T (Pharmacia LKB Biotechnology, Piscataway, NJ). Deletion of the two internal NcoI fragments from the insert of pGEX-BP180A yielded construct pGEX$\mathrm{N} \Delta 1$, encoding a GST fusion protein, designated GST-N $\Delta 1$, containing human BP180 residues 542-655 and 865-890 (see Fig. 1). A segment of the murine BP180 cDNA was amplified by the reverse transcriptase (RT) PCR (30) using mRNA from murine epidermis as the template. Since no murine BP180 sequence information was available before the initiation of these experiments, the sequences of the PCR primers (synthesized by Operon Technologies, Inc., Alameda, CA) were based on the human BP180 sequence (21), choosing stretches that were identical to the corresponding sites on the chicken BP 180 homologue (31). The positive strand primer corresponded to human BP180 nucleotide 1557-1574 and the negative strand primer to human nucleotide 2249-2234. A segment of the murine BP180 protein (amino acid residues 494-642; using the numbering system of Li et al. [32]) was expressed using the pGEX-2T system as a GST fusion protein, designated GST-mus180A (see Fig. 1). GST-N $\Delta 1$, GSTmus $180 \mathrm{~A}$, and recombinant GST (encoded by the pGEX-2T vector) were purified using previously described protocols (33).

Administration of anti-BP180 IgG to experimental animals. New Zealand White rabbits were immunized with the GST-N $\Delta 1$ and GSTmus $180 \mathrm{~A}$ fusion proteins and with recombinant GST. IgG fractions were prepared from these antisera and from preimmune rabbit sera as previously described $(26,27)$. Final IgG concentrations were measured by $\mathrm{OD}_{280}$ based on an extinction coefficient $(\mathrm{E}[1 \%, 1 \mathrm{~cm}])$ of 13.6. Neonatal BALB/c mice (12-36 h of age) were obtained from a breeding colony maintained in the laboratory and were injected intraperitoneally with the purified IgG fractions described above, following procedures reported elsewhere $(26,27)$. IgG doses ranged from 0.03 to $27 \mathrm{mg} / \mathrm{g}$ body weight. To avoid injection of excessive fluid volumes, the higher doses were given using an IgG concentration of $100 \mathrm{mg} / \mathrm{ml}$
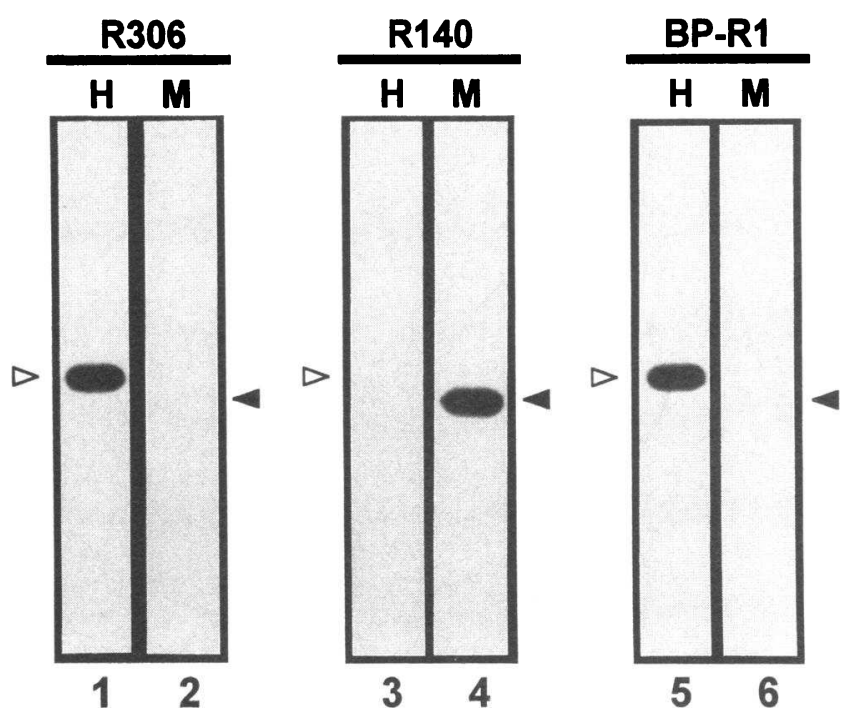

Figure 2. Characterization of the cross-species reactivity of antiBP180 antibodies. Immunoblots containing recombinant human BP180 (lanes 1,3, and 5) or murine BP180 (lanes 2, 4, and 6) were labeled with a 1:500 dilution of R306, a rabbit anti-human BP180 antiserum (lanes 1 and 2), a 1:500 dilution of R140, a rabbit anti-murine BP180 antiserum (lanes 3 and 4), or a 1:200 dilution of BP-R1, a BP patient serum (lanes 5 and 6 ). The open and filled arrowheads designate the positions of the recombinant human and murine BP180 polypeptides, respectively. R306 and BP-R1 reacted with human BP180 (lanes $l$ and 5 ) but failed to cross-react with the murine form of BP180 (lanes 2 and 6). Conversely, R140 showed reactivity with the murine form (lane 4 ) but not the human form of BP180 (lane 3). 
and the injections were given in two doses $5 \mathrm{~h}$ apart. To test for induction of localized disease, neonatal mice were given a single-dose intradermal injection of various amounts of experimental and control IgG (dissolved in $50 \mu \mathrm{l}$ of PBS) on the upper back.

Animal evaluation. Neonatal mice were examined 18 and $24 \mathrm{~h}$ after intraperitonial and intradermal injections. The extent of cutaneous disease was scored as follows: -, no detectable skin disease; $1+$, mild erythematous reaction with no evidence of the "epidermal detachment" sign (this sign was elicited by gentle friction of the mouse skin which, when positive, produced fine, persistent wrinkling of the epidermis); $2+$, intense erythema and "epidermal detachment" sign in localized areas; and $3+$, intense erythema with frank "epidermal detachment" sign involving $>20 \%$ of the epidermis.

Histological sections of lesional and perilesional skin were studied in specimens previously fixed in $10 \%$ buffered formalin and stained with hematoxylin and eosin. Direct IF of perilesional skin was performed as previously described $(26,27)$. Monospecific FITC-conjugated sera were obtained commercially: goat anti-rabbit IgG (Kirkeggard \& Perry Laboratories, Inc., Gaithersburg, MD) and monospecific goat anti-mouse C3 (Cappel Laboratories, Durham, NC).

Sera were obtained from mice at the time of biopsy and assayed for antibody titers by indirect IF using neonatal mouse skin or human skin sections as substrate $(26,27)$. Animals given control IgG fractions were studied in an identical fashion.

\section{Recuks}

Characterization of murine and human BP180 fusion proteins. Fig. 1, top, shows a schematic diagram depicting the primary structure of the human BP180 antigen based on previously published data (21). Identified on this diagram is a 14-amino acid stretch within a noncollagenous region of the BP180 ectodomain that was shown by epitope mapping analysis to be a major site of reactivity for both BP and HG autoantibodies (25). The corresponding segment of the murine form of BP180 was cloned using an RT-PCR protocol as described above. The sequence of the cloned PCR product showed a perfect match with the recently published murine BP180 cDNA sequence (32). Amino acid sequence alignment of the human and murine BP180 sequences is shown in Fig. 1, bottom. Interestingly, the well-defined BP/HG antigenic site on the human BP180 antigen (designated by the boxed-in sequence) is very poorly conserved in the murine protein. Only 3 of the 14 residues in this antigenic region are identical in these two species. This is in stark contrast to the relatively high overall homology exhibited by the human and murine BP1 180 proteins $(80.6 \%$ identity and $86 \%$ similarity [32]).

Characterization of rabbit anti-BP180 polyclonal antibodies. The antigenic specificities of the rabbit antibodies prepared against the GST-N $\Delta 1$ and GST-mus 180A fusion proteins were characterized by immunoblot analysis as shown in Fig. 2. Before analysis both the human and murine BP180 fusion proteins were digested with thrombin to separate the BP180 insert from the GST moiety. The rabbit anti-human BP180 anti- serum, R306, reacted with the human BP180 polypeptide from GST-N $\Delta 1$ but did not react with the murine BP180 homologue

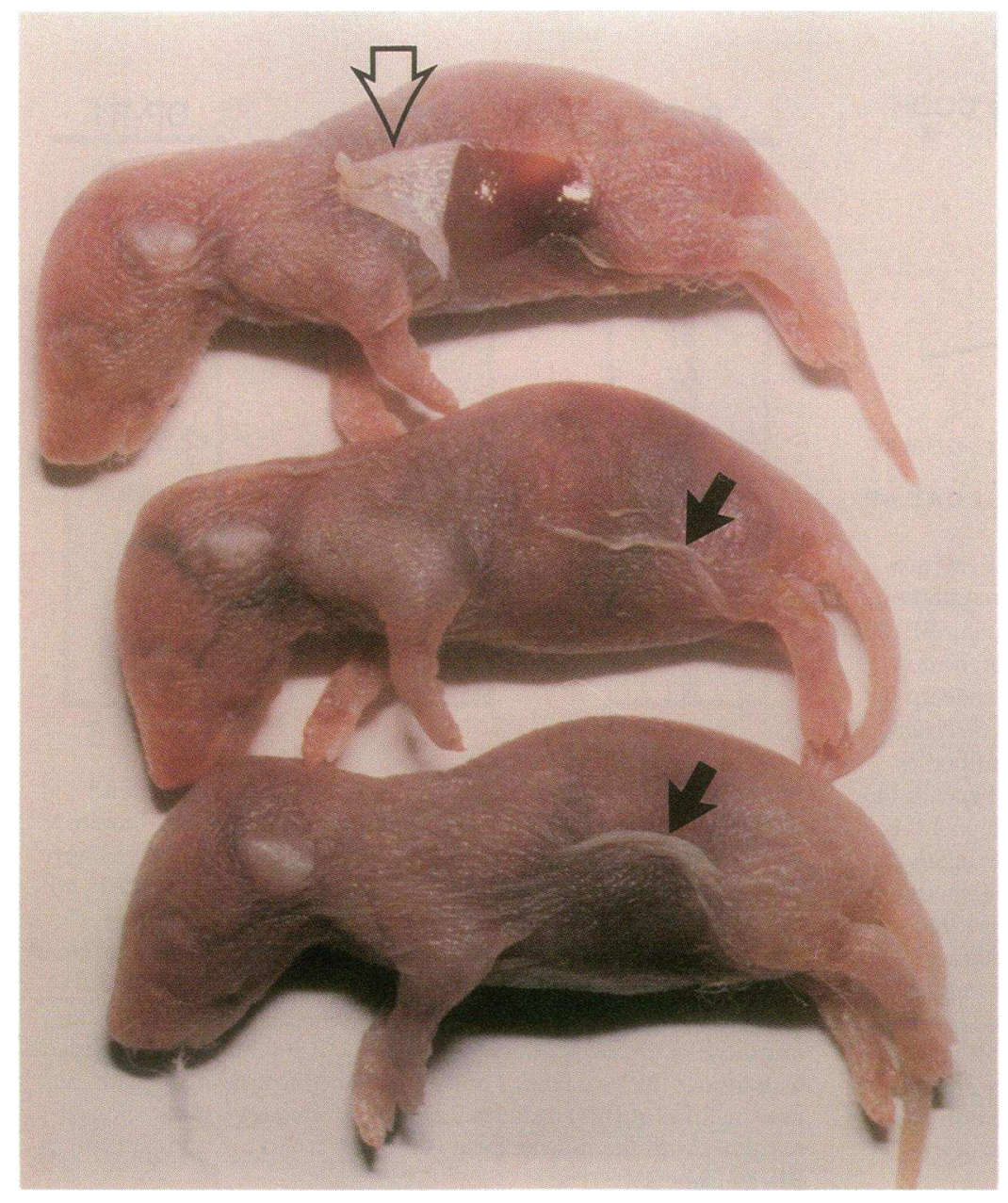

Figure 3. Neonatal BALB/c mice injected intraperitoneally with rabbit anti-murine BP180 IgG. All three animals shown received the same dose of IgG (2.7 $\mathrm{mg} / \mathrm{g}$ body weight) $18 \mathrm{~h}$ before examination. Typical "epidermal detachment" sign is observed in middle and bottom mice (filled arrow). A slit was made in the wrinkled epidermis of the top mouse and the epidermis was easily lifted away from the underlying dermis (open arrow). Mice injected with similar doses of rabbit anti-human BP180 IgG showed no skin abnormalities. 
(Fig. 2, lanes 1 and 2). Conversely, the rabbit anti-murine BP180 antiserum, R140, reacted with the murine but not the human form of BP180 (Fig. 2, lanes 3 and 4). Significantly, a BP serum, which previously had been shown to lack pathogenic IgG activity in the passive transfer mouse model (28), showed reactivity with recombinant human $\mathrm{BP} 180$ but did not crossreact with the murine form of BP180 (Fig. 2, lanes 5 and 6).

Clinical evaluation of neonatal mice injected with $\operatorname{Ig} G$ fractions. Neonatal BALB/c mice (12-36 h of age) were given intraperitoneal or intradermal injections of purified IgG fractions prepared from the rabbit anti-human BP180 and rabbit anti-murine BP1 180 antisera and control rabbit sera. $24 \mathrm{~h}$ after intraperitoneal injection of rabbit anti-murine BP $180 \mathrm{IgG}$ into mice the skin of the injected animals was markedly erythematous and, upon gentle friction, developed persistent epidermal wrinkling producing the "epidermal detachment" sign described in Methods (Fig. 3). This finding resembled the "Nikolsky" sign induced in the skin of neonatal mice injected with either pemphigus vulgaris or pemphigus foliaceus $\operatorname{IgG}(26$, 27). In contrast to skin showing the typical Nikolsky sign, the "epidermal detachment" induced by the IgG from the rabbit anti-murine BP180 antiserum was resistant to erosion by friction. After slitting the lesional epidermal wrinkle of these animals, an epidermal sheet could be easily lifted away, exposing the underlying dermis (Fig. 3, top). The severe cutaneous signs induced by the rabbit anti-murine BP180 IgG were dose dependent. Mice injected with doses of $\geq 2.7 \mathrm{mg} / \mathrm{g}$ body weight $(n=7)$ developed extensive $(3+)$ disease. Animals injected with lower doses $(270 \mu \mathrm{g} / \mathrm{g}$ body weight $[n=7]$ and $27 \mu \mathrm{g} / \mathrm{g}$ body weight $[n=7]$ ) exhibited minimal $(1+)$ reaction or no reaction, respectively. The skin of neonatal mice given intraper-

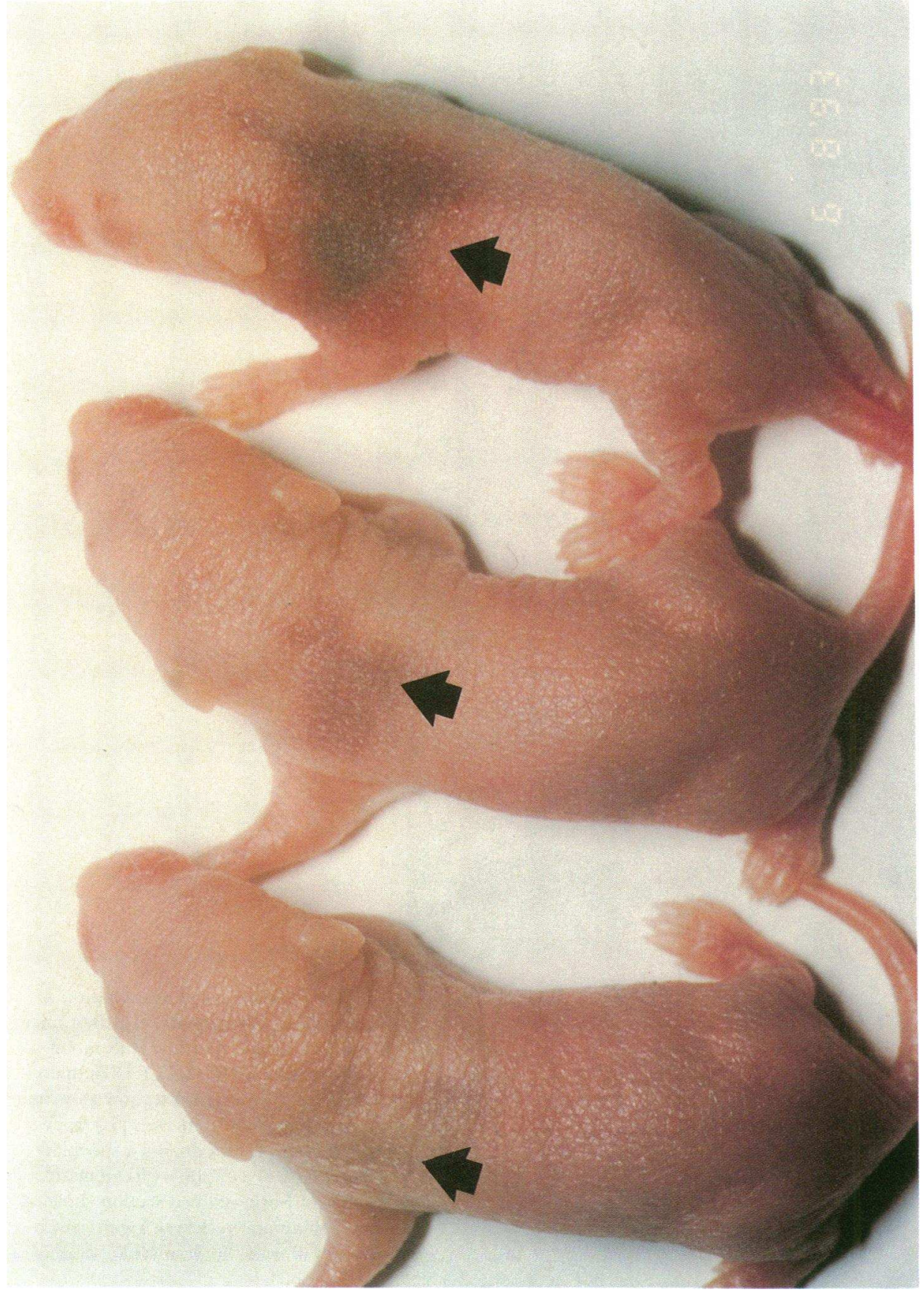

Figure 4. Neonatal BALB/c mice injected intradermally in the upper back with rabbit anti-murine BP180 IgG. The mice shown at the top, middle, and bottom received 13.5 $\mathrm{mg}, 1.35 \mathrm{mg}$, and $135 \mu \mathrm{g} \mathrm{IgG} / \mathrm{g}$ body weight, respectively, in a $50 \mu \mathrm{l}$ volume $18 \mathrm{~h}$ before evaluation. The arrows on the mice shown at the top and middle point to the areas of inflammation triggered by the rabbit IgG measuring 8 and $4 \mathrm{~mm}$ in diameter, respectively. No inflammatory reaction was observed in the animal at the bottom. Identical doses of rabbit anti-human BP180 IgG did not produce any detectable inflammatory changes in the injected animals. 
itoneal injections of IgG from preimmune rabbit $\mathrm{R} 140$ serum $(n=5)$, rabbit anti-human BP180 serum $(n=5)$, or rabbit anti-GST serum $(n=3)$ was completely normal.

Intradermal injections of IgG isolated from the rabbit antimurine BP180 serum induced a localized inflammatory reaction at the injection site (Fig. 4). Injections of $13.5 \mathrm{mg} \mathrm{IgG/g}$ body weight $(n=2)$ triggered an acute inflammatory response (2+ reaction) that involved an area $8 \mathrm{~mm}$ in diameter (Fig. 4, top). The erythematous reaction was less intense ( $1+$ reaction) and smaller in diameter $(4 \mathrm{~mm})$ at sites injected with $1.35 \mathrm{mg}$ IgG/g body weight $(n=4)$ (Fig. 4, middle), and was absent at sites injected with $135 \mu \mathrm{g} \mathrm{IgG/g}$ body weight $(n=2)$ (Fig. 4, bottom).

Histological evaluation of neonatal mice injected with IgG fractions. Histological examination of the skin of mice injected intraperitoneally with rabbit anti-murine BP180 IgG demon- strated broad subepidermal vesicle formation. In most zones there were small numbers of inflammatory cells in the subepidermal clefts or underlying dermis (Fig. $5 \mathrm{~A}$ ). In focal zones, especially near the edges of the blisters, neutrophils were present in large numbers within the blister cavity and were scattered in smaller numbers in the nearby dermis (Fig. 5 B). In only very limited zones, increased numbers of mast cells accompanied the neutrophils. Necrotic keratinocytes were only infrequently observed in the epidermis or roofs of vesicles, and there was no evidence of vasculitis. These changes were most evident in mice injected with a concentration of $2.7 \mathrm{mg} / \mathrm{g}$ body weight of IgG. In mice injected with $270 \mu \mathrm{g} / \mathrm{g}$ body weight of $\mathrm{IgG}$, focal hints of subepidermal separation were identified without blister formation, and in the nearby dermis inflammation with scattered neutrophils and mast cells was present. The skin of mice injected intraperitoneally with rabbit anti-human IgG
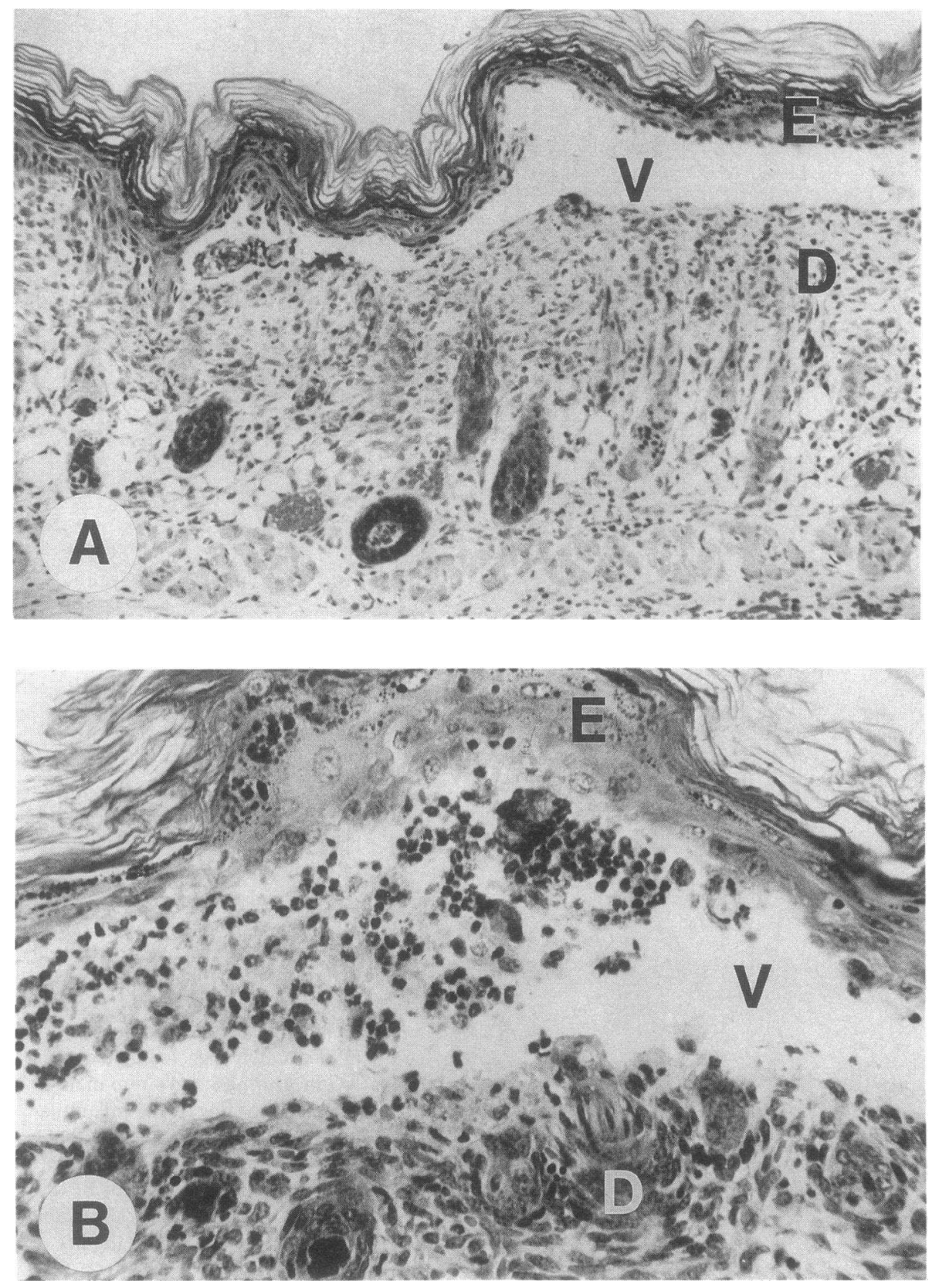

Figure 5. Histological examination of lesional skin of neonatal BALB/c mice injected intraperitoneally with rabbit anti-murine BP180 IgG. $(A)$ Hematoxylin and Eosin-stained section showing separation of the epidermis $(E)$ from the dermis $(D)$ producing a subepidermal vesicle $(V)(\times 300)$. (B) Hematoxylin and Eosin-stained section showing a subepidermal vesicle $(V)$ with neutrophilic infiltrate. Epidermis $(E)$; dermis (D) $(\times 600)$. 
did not develop any clinical signs in their skin, and histological exam demonstrated normal epidermis and dermis without any pathological alterations.

Local intradermal injection of rabbit anti-murine BP180 IgG produced at sites of clinical reaction histological changes identical to those of intraperitoneally injected animals with blister formation and focal inflammation containing neutrophils. The skin of mice injected intradermally with anti-human BP180 IgG was normal.

Immunological evaluation of neonatal mice injected intraperitoneally with IgG fractions. Table I shows the results of a representative set of experiments in which three pairs of animals each received intraperitoneal injections with different doses of anti-murine BP180 IgG. These data clearly show that increasing the amount of injected IgG resulted in an increase in the circulating titer of mouse-specific anti-BP180 antibody, which, in turn, was associated with increased cutaneous disease activity. Mice injected with the lowest dose of IgG (27 $\mu \mathrm{g} / \mathrm{g}$ body weight) did not have detectable levels of circulating rabbit anti-murine BP180 IgG nor did they show signs of skin disease, although low levels of tissue-bound rabbit IgG and mouse complement were detected at the cutaneous BMZ of these animals (Fig. 6, $A$ and $B$ ). Extensive disease ( $3+)$ and high titers of circulating anti-murine BMZ antibodies $(1: 160)$ were observed in animals treated with a high dose of rabbit anti-murine BP180 IgG $(2.7 \mathrm{mg} / \mathrm{g}$ body weight $)$, but detection of in situ bound immunoreactants was variable and appeared to be site dependent in these animals (IgG was detected at low levels in nonlesional but not at all in lesional skin; $\mathrm{C} 3$ was only seen in lesional skin and then in only one of two animals). Animals given an intermediate dose of rabbit anti-murine BP180 IgG ( $270 \mu \mathrm{g} / \mathrm{g}$ body weight) showed intermediate levels of circulating IgG titers $(1: 80)$ and disease activity $(1+)$ and variable levels of BMZ-associated immunoreactants.

To more accurately determine the location of the split induced in the skin of mice injected with a high dose of anti-murine BP180 IgG, lesional skin cryosections were labeled by indirect IF with a human BP serum. This patient serum had previously been shown to react with both BP230 and BP180 on immunoblots and with the BMZ of both human and murine skin by indirect IF (data not shown). As shown in Fig. $6 C$, the human BP autoantibodies exhibited linear staining of the epidermal side of the vesicle in the mouse skin, a reactivity pattern identical to that detected in lesional skin of human BP patients.
Comparison of the data in Tables I and II reveals a relationship between species-restricted reactivity and the pathogenic activity of the two anti-BP1 80 antibody preparations. Mice injected with anti-human BP180 IgG $(0.27$ and $2.7 \mathrm{mg} / \mathrm{g}$ body weight) developed high titers of circulating antibody (1:160640 ) when assayed by indirect IF against normal human skin. But, in accordance with the lack of crossreactivity of this antibody with murine BP180 demonstrated by immunoblot (Fig. 2 ), mice injected with anti-human BP180 antibody showed no circulating or tissue-bound anti-murine BMZ reactivity and exhibited no pathological effects.

Table III shows the results of a representative experiment in which three different concentrations of rabbit anti-murine BP180 IgG were injected intradermally into neonatal mice. Similar to the dose response observed with the intraperitoneal injections, an increase in the dose of anti-murine BP180 IgG given intradermally resulted in an increase in the titer of antimurine BMZ antibodies detected in the serum of the injected mice and in an increase in local skin disease activity.

\section{Discussion}

Several independent lines of evidence derived from clinical, histologic, and immunologic investigations have implicated the autoimmune response as a key element in the pathogenesis of BP and HG (3-6). However, previous attempts to directly demonstrate the pathogenic activity of autoantibodies produced by these patients using systemic passive transfer techniques have been largely unsuccessful $(28,29)$. This was apparently due, as shown in this paper, to a lack of crossreactivity of the pathogenic autoantibodies with the autoantigen homologue of the experimental host animal. In the present investigation we have succeeded in generating antibodies to the murine BP1 180 protein that, when passively transferred (intradermally or intraperitoneally) into neonatal BALB/c mice, induced a cutaneous blistering disease that faithfully reproduced all of the immunopathological features that are the hallmark of the human diseases, BP and HG (34).

The skin lesions induced by the anti-murine BP180 IgG were characterized by an "epidermal detachment" sign, i.e., fine, persistent wrinkling of the mouse epidermis produced by mild friction (Fig. 3). Histological examination of the mouse skin showed subepidermal vesicles and a rich neutrophilic in-

Table I. Mice Injected Intraperitoneally with Anti-murine BP180 IgG

\begin{tabular}{|c|c|c|c|c|c|c|}
\hline \multirow[b]{2}{*}{ Mouse no. } & \multirow[b]{2}{*}{ IgG dose } & \multirow[b]{2}{*}{ Disease } & \multicolumn{2}{|c|}{ Direct IF } & \multicolumn{2}{|c|}{ Indirect IF } \\
\hline & & & $\operatorname{IgG}$ & $\mathrm{C} 3$ & Mouse skin & Human skin \\
\hline \multirow[t]{2}{*}{1 (R5A1) } & $2.7 \mathrm{mg}$ & +++ & Nonlesion (+) & $(-)$ & $1: 640$ & $(-)$ \\
\hline & & & Lesion (-) & $(+)$ & & \\
\hline \multirow[t]{2}{*}{2 (R5A2) } & $2.7 \mathrm{mg}$ & +++ & Nonlesion (+) & $(-)$ & $1: 320$ & $(-)$ \\
\hline & & & Lesion (-) & $(-)$ & & \\
\hline \multirow[t]{2}{*}{3 (R5B1) } & $270 \mu \mathrm{g}$ & + & Nonlesion (-) & $(-)$ & $1: 80$ & $(-)$ \\
\hline & & & Lesion (-) & $(-)$ & & \\
\hline \multirow[t]{2}{*}{4 (R5B2) } & $270 \mu \mathrm{g}$ & + & Nonlesion $(+)$ & $(-)$ & $1: 80$ & $(-)$ \\
\hline & & & Lesion (+) & $(+)$ & & \\
\hline $5(\mathrm{R} 5 \mathrm{Cl})$ & $27 \mu \mathrm{g}$ & $(-)$ & Nonlesion (+) & $(+)$ & $(-)$ & $(-)$ \\
\hline $6(\mathrm{R} 5 \mathrm{C} 2)$ & $27 \mu \mathrm{g}$ & $(-)$ & Nonlesion (+) & $(+)$ & $(-)$ & $(-)$ \\
\hline
\end{tabular}



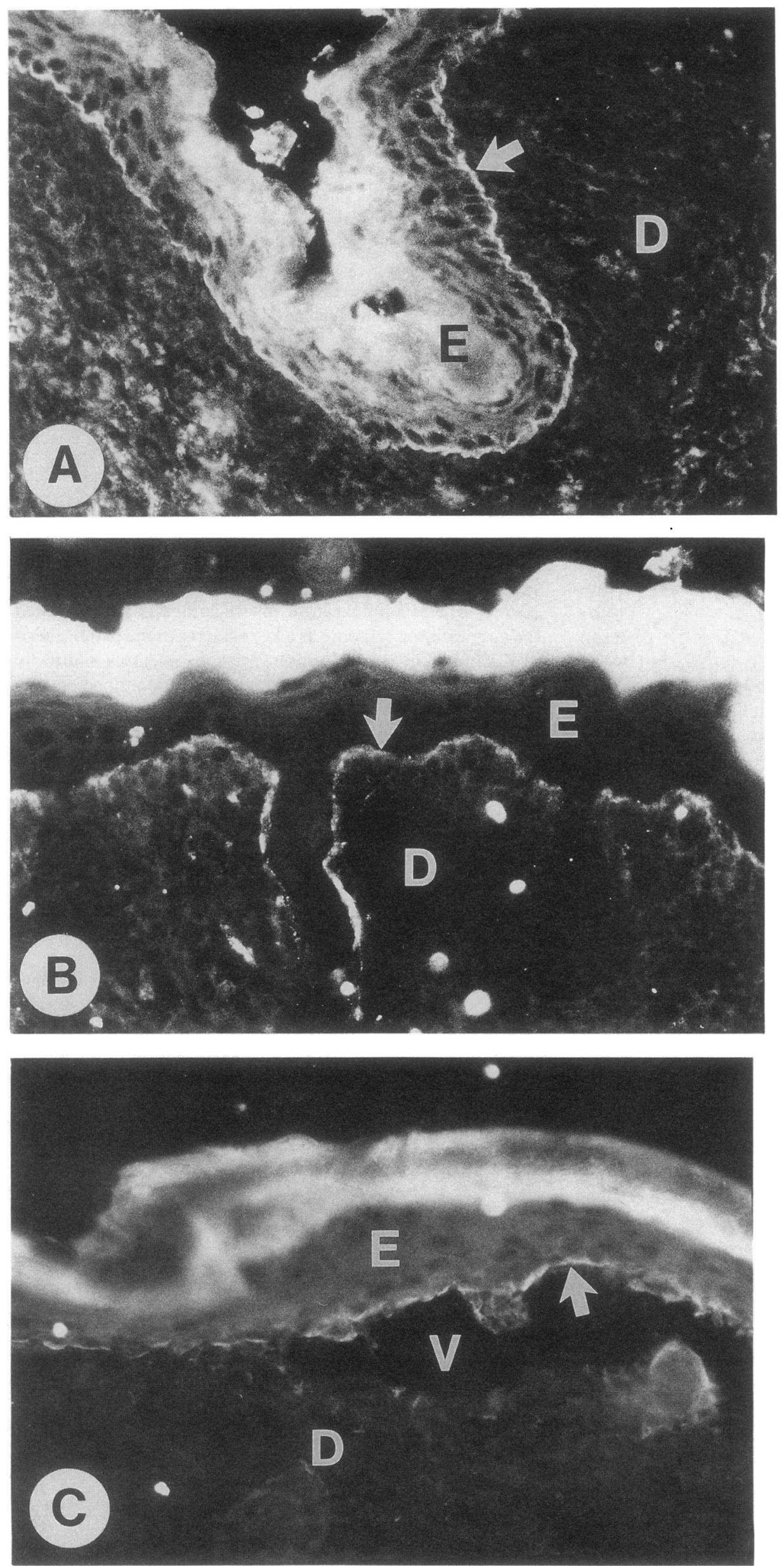

Figure 6. Immunofluorescence analysis of perilesional skin of neonatal BALB/ $\mathrm{c}$ mice injected intraperitoneally with rabbit anti-murine BP180 IgG. ( $A$ and $B)$ Cryosections of mouse skin treated with FITC-goat anti-rabbit IgG and FITC-goat anti-mouse $\mathrm{C} 3$, respectively $(\times 400)$. In both cases linear staining of the BMZ was observed (arrows). (C) Lesional mouse skin cryosection incubated with a serum from a bullous pemphigoid patient for the purpose of mapping the site of cleavage of the BMZ. Bound BP autoantibodies were visualized using an FITC-goat antihuman IgG and produced a linear staining of the intact BMZ (arrow) and the epidermal roof of the vesicle $(V)$. Epidermis $(E)$, dermis $(D)(\times 400)$. 
Table II. Mice Injected Intraperitoneally with Anti-human BP180 IgG

\begin{tabular}{|c|c|c|c|c|c|c|}
\hline \multirow[b]{2}{*}{ Mouse no. } & \multirow[b]{2}{*}{ IgG dose } & \multirow[b]{2}{*}{ Disease } & \multicolumn{2}{|c|}{ Direct IF } & \multicolumn{2}{|c|}{ Indirect IF } \\
\hline & & & IgG & $\mathrm{C} 3$ & Mouse skin & Human skin \\
\hline \multirow[t]{3}{*}{1 (R306A) } & $2.7 \mathrm{mg}$ & $(-)$ & Head (-) & $(-)$ & $(-)$ & $1: 640$ \\
\hline & & & Shoulder (-) & $(-)$ & & \\
\hline & & & Hip (-) & $(-)$ & & \\
\hline \multirow[t]{3}{*}{2 (R306B) } & $2.7 \mathrm{mg}$ & $(-)$ & Head (-) & $(-)$ & $(-)$ & $1: 640$ \\
\hline & & & Shoulder (-) & $(-)$ & & \\
\hline & & & $\operatorname{Hip}(-)$ & $(-)$ & & \\
\hline \multirow[t]{3}{*}{3 (R306C) } & $270 \mu \mathrm{g}$ & $(-)$ & Head (-) & $(-)$ & $(-)$ & $1: 160$ \\
\hline & & & Shoulder (-) & $(-)$ & & \\
\hline & & & Hip (-) & $(-)$ & & \\
\hline
\end{tabular}

filtrate in the upper dermis that, in certain areas, abutted the dermal-epidermal junction. BP autoantibodies reacted by indirect IF with the roof of the vesicle in the mouse skin (Fig. $6 C$ ), as observed in lesional skin of human BP patients. C3 was the immunoreactant most consistently detected in the perilesional skin of mice injected with anti-murine BP180 IgG (Fig. $6 \mathrm{~B}$ ). Linear deposition of rabbit $\mathrm{IgG}$ at the $\mathrm{BMZ}$ of perilesional skin was also detected in mice injected with a low dose of anti-murine BP1 $80 \mathrm{IgG}(27 \mu \mathrm{g} / \mathrm{g}$ body weight) (Fig. $6 \mathrm{~A}$ ), but was not always seen in mice injected with higher doses $(0.27-27 \mathrm{mg} / \mathrm{g}$ body weight). The higher doses of IgG produced more extensive inflammation and vesiculation in the injected animals, which may, in turn, account for destruction of tissue-bound IgG. Similarly, BMZ deposition of IgG is often not detected in perilesional skin of BP patients with extensive disease, nor in the skin of moderately affected BP patients within or immediately adjacent to lesional areas.

The sera of the host animals used in the passive transfer experiments (including both test and control groups) were assayed by indirect IF and revealed antibody activities with substrate specificities corresponding to those of the injected IgG. Mice injected intraperitoneally with high doses of rabbit antimurine BP180 IgG developed high circulating anti-BMZ titers when assayed against normal mouse skin. The sera of mice injected intraperitoneally with high doses of rabbit anti-human BP180 IgG contained high indirect IF titers when using human skin as substrate, but showed no reactivity with mouse skin. Mice injected with preimmune rabbit IgG or IgG from a rabbit anti-GST serum showed no circulating antibody reactivity with either human or mouse skin, as expected. The extent of disease induced in mice by rabbit anti-murine BP180 IgG showed a direct correlation with the titers of antibodies detected in the sera of the injected mice; e.g., mice with circulat- ing anti-murine $\mathrm{BMZ}$ antibody titers $\geq 1: 160$ exhibited extensive skin disease.

Neonatal mice injected intradermally with rabbit antimurine BP180 IgG also developed circulating antibody titers that showed a direct correlation both with the amount of $\mathrm{IgG}$ injected and with the extent of the inflammatory response seen at the injection sites. Visual examination (Fig. 4) of the mice injected intradermally with high doses of this IgG showed intense dose-dependent erythema and subepidermal vesicles at the microscopic level. It is likely that repeated intradermal injections using high doses of the anti-murine BP180 IgG would precipitate skin disease in regions distant from the intradermal injection sites. The specificity of the effects elicited by the local intradermal injections was demonstrated by testing IgG from control sera that did not induce skin disease in the experimental animals.

Several investigators have previously attempted to induce cutaneous blistering eruptions in experimental animals by intradermal injection of BP serum or its IgG fraction $(35,36)$. These studies were inconclusive due to the variability of the responses observed in the experimental animals. For example, Naito et al. (35) found that intradermal injections of BP serum or BP IgG into guinea pigs was associated with dermal-epidermal separation mediated by neutrophils and complement. Gammon and Briggaman (36), however, could not reproduce these findings. Our laboratory demonstrated that local intrastromal injections of BP IgG into rabbit corneas induced separation of the epithelium from the stroma and migration of neutrophils into the epithelial-stromal junction (37). Contralateral corneas injected with normal human IgG showed no inflammatory changes. The effects produced by the human IgG on the rabbit cornea were dose dependent and appeared to be mediated by activation of complement. The interpretation

Table III. Mice Injected Intradermally with Anti-murine BP180 IgG

\begin{tabular}{|c|c|c|c|c|c|c|}
\hline \multirow[b]{2}{*}{ Mouse no. } & \multirow[b]{2}{*}{ IgG dose } & \multirow[b]{2}{*}{ Disease } & \multicolumn{2}{|c|}{ Direct IF } & \multicolumn{2}{|c|}{ Indirect IF } \\
\hline & & & IgG & $\mathrm{C} 3$ & Mouse skin & Human skin \\
\hline 1 (R5D3) & $13.5 \mathrm{mg}$ & ++ & $\begin{array}{l}\text { Intradermal site }(-) \\
\text { Nonlesion }(-)\end{array}$ & $\begin{array}{l}(+) \\
(+)\end{array}$ & $1: 1280$ & $(-)$ \\
\hline 2 (R5D2) & $1.35 \mathrm{mg}$ & + & $\begin{array}{l}\text { Intradermal site }(-) \\
\text { Nonlesion }(-)\end{array}$ & $\begin{array}{l}(-) \\
(-)\end{array}$ & $1: 80$ & $(-)$ \\
\hline 3 (R5D1) & $135 \mu \mathrm{g}$ & $(-)$ & $\begin{array}{l}\text { Intradermal site }(+) \\
\text { Nonlesion }(-)\end{array}$ & $\begin{array}{l}(-) \\
(+)\end{array}$ & $1: 40$ & $(-)$ \\
\hline
\end{tabular}


of these earlier studies must be reevaluated in light of current information regarding the heterogeneous nature of the antigen/antibody systems present in BP sera.

In summary, this study shows that polyclonal rabbit antibodies raised against an extracellular noncollagenous domain of the murine BP180 antigen are pathogenic when introduced into neonatal mice. The subepidermal vesiculation caused by these antibodies was always associated with $\mathrm{C} 3$ deposition at the BMZ and infiltration of the upper dermis with neutrophils, suggestive of a complement-mediated pathogenic mechanism. The murine BP180 protein segment that triggered the production of pathogenic rabbit antibodies corresponds to a region on the human BP180 homologue that is recognized by a high percentage of both BP and HG patients' sera (25). Thus, it is highly likely that blister formation in BP and HG patients is mediated by human autoantibodies directed against this welldefined immunodominant epitope, either via a complementactivation and neutrophilic injury mechanism or by directly disrupting the function of the BP180 protein. The experimental model of BP/HG described in this paper will undoubtedly be a very useful tool in dissecting the molecular and immunological mechanisms of subepidermal blister formation in BP and $\mathrm{HG}$, and in developing more effective therapeutic strategies for managing these autoimmune disorders.

\section{Acknowledgments}

This work was supported in part by U.S. Public Health Service grants R29 AR-40410 (G. J. Giudice), and R01 AR-32599 and R37AR32081 (L. A. Diaz) from the National Institutes of Health and by a VA Merit Review Grant (L. A. Diaz). Z. Liu is the recipient of a Burroughs Wellcome Fund Research Fellowship awarded by the Dermatology Foundation.

\section{References}

1. Lever, W. F. 1953. Pemphigus. Medicine. 32:1-123.

2. Shornick, J. K., J. L. Bangert, R. G. Freeman, and J. N. Gilliam. 1983. Herpes gestationis: clinical and histological features of twenty-eight cases. J. Am. Acad. Dermatol. 8:214-224.

3. Jordon, R. E., E. H. Beutner, E. Witebsky, G. Blumental, W. C. Hale, and W. F. Lever. 1967. Basement zone antibodies in bullous pemphigoid. J. Am. Med. Assoc. 200:751-758.

4. Provost, T. T., and T. B. Tomasi, Jr. 1973. Evidence for complement activation via the alternative pathway in skin diseases. I. Herpes gestationis, systemic lupus erythematosus, and bullous pemphigoid. J. Clin. Invest. 52:17791787.

5. Jordon, R. E., K. G. Heine, G. Tappeiner, L. L. Bushkell, and T. T. Provost. 1976. The immunopathology of herpes gestationis. Immunofluorescent studies and characterization of the "HG factor." J. Clin. Invest. 57:1426-1433.

6. Katz, S. I., K. C. Hertz, and H. Yaoita. 1976. Herpes gestationis. Immunopathology and characterization of the HG factor. J. Clin. Invest. 57:1434-1441.

7. Schaumburg-Lever, G., A. Rule, and B. Schmidt-Ulrich. 1975. Ultrastructural localization of in vivo bound immunoglobulins in bullous pemphigoid: a preliminary report. J. Invest. Dermatol. 64:47-49.

8. Karpati, S., W. Stolz, M. Meurer, O. Braun-falco, and T. Krieg. 1991. Herpes gestationis: Ultrastructural identification of the extracellular antigenic sites in diseased skin using immunogold techniques. Br. J. Dermatol. 125:317324.

9. Kelly, S. E., R. Cerio, B. S. Bhogal, and M. M. Black. 1989. The distribution of IgG subclasses in pemphigoid gestationis: PG Factor is an IgG1 autoantibody. J. Invest. Dermatol. 92:695-698.

10. Suzuki, M., S. Harada, and Y. Yaoita. 1992. Purification of bullous pemphigoid IgG subclasses and their capability for complement fixation. Acta Dermatovenereol. 72:245-249.

11. Chorzelski, T. P., S. Jablonska, E. H. Beutner, E. Maciejowska and J. Jarzabek-Chorzelska. 1976. Herpes gestationis with identical lesions in the newborn. Arch. Dermatol. 112:1129-1131.

12. Katz, A., J. O. Minta, J. W. P. Toole, and W. Medwidsky. 1977. Immunopathologic study in herpes gestationis in mother and child. Arch. Dermatol. 113:1069-1072.

13. Carruthers, J. A., and A. R. Ewins. 1978. Herpes gestationis: studies on the binding characteristics, activity and pathogenetic significance of the complement-fixing factor. Clin. Exp. Immunol. 31:38-44.

14. Van de Wiel, A., H. Hart, J. Flinterman, J. A. M. Kerckhaert, J. A. Du Boeuff, and J. W. Imhof. 1980. Plasma exchange in herpes gestationis. Br. Med. J. 281:1041-1042.

15. Labib, R. S., G. J. Anhalt, H. P. Patel, D. F. Mutasim, and L. A. Diaz 1986. Molecular heterogeneity of bullous pemphigoid antigens as detected by immunoblotting. J. Immunol. 136:1231-1235.

16. Morrison, L. H., R. S. Labib, J. J. Zone, L. A. Diaz, and G. J. Anhalt. 1988. Herpes gestationis autoantibodies recognize a $180-\mathrm{kD}$ human epidermal antigen. J. Clin. Invest. 81:2023-2026.

17. Stanley, J. R., T. Tanaka, S. Mueller, V. Klaus-Kovtun, and D. Roop. 1988. Isolation of a complementary DNA for bullous pemphigoid antigen by use of patients' autoantibodies. J. Clin. Invest. 82:1864-1870.

18. Diaz, L. A., H. Ratrie III, W. S. Saunders, S. Futamura, H. L. Squiquera, G. J. Anhalt, and G. J. Giudice. 1990. Isolation of a human epidermal cDNA corresponding to the $180-\mathrm{kD}$ autoantigen recognized by bullous pemphigoid and herpes gestationis sera. Immunolocalization of this protein to the hemidesmosome. J. Clin. Invest. 86:1088-1094.

19. Mutasim, D. F., Y. Takahashi, R. S. Labib, G. J. Anhalt, H. P. Patel, and L. A. Diaz. 1985. A pool of bullous pemphigoid antigen(s) is intracellular and associated with the basal cell cytoskeleton-hemidesmosome complex. J. Invest. Dermatol. 84:47-53.

20. Tanaka, T., N. J. Korman, H. Shimizu, A. J. Eady, V. Klaus-Kovtun, K. Cehrs, and J. R. Stanley. 1990. Production of rabbit antibodies against carboxyterminal epitopes encoded by bullous pemphigoid cDNA. J. Invest. Dermatol. 94:617-623.

21. Giudice, G. J., D. J. Emery, and L. A. Diaz. 1992. Cloning and primary structural analysis of the bullous pemphigoid autoantigen, BP-180. J. Invest. Dermatol. 99:243-250.

22. Hopkinson, S. B., K. S. Riddelle, and J. C. R. Jones. 1992. Cytoplasmic domain of the $180-\mathrm{kD}$ bullous pemphigoid antigen, a hemidesmosomal component: molecular and cell biologic characterization. J. Invest. Dermatol. 99:264270.

23. Giudice, G. J., H. L. Squiquera, P. M. Elias, and L. A. Diaz. 1991. Identifcation of two collagen domains within the bullous pemphigoid autoantigen, BP180. J. Clin. Invest. 87:734-738.

24. Nishikawa, Y., J. Uematsu, and K. Owaribe. 1993. HD4, a 180kDa bullous pemphigoid antigen, is a major transmembrane glycoprotein of the hemidesmosome. J. Biochem. 113:493-501.

25. Giudice, G. J., D. J. Emery, B. D. Zelickson, G. J. Anhalt, Z. Liu and L. A. Diaz. 1993. Bullous pemphigoid and herpes gestationis autoantibodies recognize a common non-collagenous site on the BP180 ectodomain. J. Immunol. In press.

26. Anhalt, G. J., R. S. Labib, J. J. Voorhees, T. F. Beals, and L. A. Diaz. 1982. Induction of pemphigus in mice by passive transfer of $\mathrm{IgG}$ from patients with the disease. N. Engl. J. Med. 306:1189-1196.

27. Roscoe, J. T., L. A. Diaz, S. A. P. Sampaio, R. M. Castro, R. S. Labib, H. Patel, and G. J. Anhalt. 1985. Brazilian pemphigus foliaceus autoantibodies are pathogenic to BALB/c mice by passive transfer. J. Invest. Dermatol. 85:538-541.

28. Anhalt, G. J., and L. A. Diaz. 1987. Animals models for bullous pemphigoid. Clin. Dermatol. 5:117-125.

29. Sams, W. M. Jr, and G. J. Gleich. 1971. Failure to transfer bullous pemphigoid with serum of patients. Proc. Soc. Exp. Biol. Med. 136:1027-1031.

30. Kawasaki, E. S., and A. M. Wang. 1989. Detection of gene expression. In PCR Technology. H. A. Erlich, editor. Stockton Press, New York. 89-97.

31. Marchant, J. K., T. F. Linsenmayer, and M. K. Gordon. 1991. cDNA analysis predicts a cornea-specific collagen. Proc. Natl. Acad. Sci. USA. 88:1560 1564.

32. Li, K., K. Tamai, E. M. L. Tan, and J. Uitto. 1993. Cloning of type XVII collagen. Complementary and genomic DNA sequences of mouse 180-Kilodalton bullous pemphigoid antigen (BPAG2) predict an interrupted collagenous domain, a transmembrane segment, and unusual features in the 5'-end of the gene and the 3'-untranslated region of the mRNA. J. Biol. Chem. 268:88258834.

33. Liu, Z., L. A. Diaz, A. L. Haas, and G. J. Giudice. 1992. cDNA cloning of a novel human ubiquitin carrier protein. An antigenic domain specifically recognized by endemic pemphigus foliaceus autoantibodies is encoded in a secondary reading frame of this human epidermal transcript. J. Biol. Chem. 267:1582915835.

34. Morrison, L. H., L. A. Diaz, G. J. Anhalt. 1990. Bullous pemphigoid. In Management of Blistering Diseases. F. Wojnarowska, and R. A. Briggaman, editors. Chapman and Hall, Inc., New York. 63-82.

35. Naito, K., S. Morioka, S. Ikeda, and H. Ogawa. 1984. Experimental bullous pemphigoid in guinea pigs: the role of pemphigoid antibodies, complement, and migrating cells. J. Invest. Dermatol. 82:227-230.

36. Gammon, W. R., and R. A. Briggaman. 1988. Absence of specific histologic changes in guinea pig skin treated with bullous pemphigoid antibodies. $J$. Invest. Dermatol. 90:495-500.

37. Anhalt, G. J., C. F. Bahn, R. S. Labib, J. J. Voorhees, A. Sugar, and L. A. Diaz. 1981. Pathogenic effects of bullous pemphigoid autoantibodies on rabbit corneal epithelium. J. Clin. Invest. 68:1097-1101. 